\title{
Cerebral Venous Thrombosis: Reviewing Pathophysiology, Diagnosis, and Treatment Strategies
}

\author{
${ }^{1}$ Department of Neurology, Duke University Hospital, DUMC, \\ Durham, North Carolina, United States \\ 2Department of Radiology, Duke University Hospital, DUMC, \\ Durham, North Carolina, United States \\ ${ }^{3}$ Department of Anesthesiology and Neurology, Duke University, \\ Durham, North Carolina, United States
}

Yasmin A. O’Keefe ${ }^{1}$ Peter G. Kranz ${ }^{2} \quad$ Keith E. Dombrowski ${ }^{1} \quad$ Brad J. Kolls ${ }^{1} \quad$ Michael L. James $^{3}$

\begin{abstract}
Address for correspondence Yasmin A. O'Keefe, MD, Department of Neurology, Duke University Hospital, DUMC Box 3824, Durham, NC 27710, United States (e-mail: yasmin.ali@duke.edu).
\end{abstract}

J Neuroanaesthesiol Crit Care 2019;6:140-144

\begin{abstract}
Keywords

- cerebral venous thrombosis

- anticoagulation

- venous hemorrhage

This review discusses cerebral venous thrombosis (CVT), including diagnosis and treatment strategies, a rare class of stroke that, if unrecognized or untreated, can have devastating effects. Thrombosis of one or many cerebral veins leads to propagation of thrombosis and impaired cerebral venous drainage. Diagnosis is made using a combination of history and imaging, particularly computed tomography (CT) venogram, which demonstrates thrombosis. Currently, acute treatment consists of heparin infusion with transition to long-term oral anticoagulation. Further research, especially on prevention, endovascular therapy, and the role of newer anticoagulants (direct oral anticoagulants [DOACs]) is necessary and ongoing.
\end{abstract}

\section{Introduction}

Cerebral venous thrombosis (CVT) is a rare subclass of strokes involving thrombosis of major intracranial veins. Incidence is estimated to be 0.2 to 1.5 in 100,000 persons. ${ }^{1}$ However, these numbers may be an underrepresentation of the true incidence, as CVT can be an elusive diagnosis and often goes unrecognized. Susceptible populations include those with prothrombotic states, either intrinsic or acquired. Clinical features of this syndrome include headache, focal neurological deficits, seizures, coma, and death. Headache is ubiquitous but variable in quality from progressive and insidious to sudden and severe (so-called thunderclap headache).

\section{Pathophysiology}

Cerebral venous thrombosis affects the main venous anatomy of the brain, resulting in thrombosis of one or more vessels. Considering Virchow's triad ${ }^{2}$ (i.e., blood stasis/nonlaminar flow, hypercoagulability/abnormal hemostasis, and endothelial injury, as shown in - Fig. 1), one or more of these factors contribute to venous thrombosis of cerebral vessels. Often beginning in a dural venous sinus, such as the transverse or superior sagittal sinus or isolated cortical veins, ${ }^{1,3}$ thrombosis propagates into surrounding veins and those draining deeper structures resulting in potentially devastating symptoms. Thrombosis of the deeper venous structures, such as inferior sagittal sinus, can compromise drainage and perfusion of important subcortical brain structures (thalami, deep nuclear structures, and brainstem) with resultant coma or even death. ${ }^{1}$

\section{Clinical Features}

Clinical presentation of CVT can be quite variable, but headache is ubiquitous, due to direct dural irritation and increased intracranial pressure (ICP). Papilledema can be visualized if the disease is particularly long standing or advanced. Development of the headache can be insidious or rapid and intense, so-called thunderclap headache, ${ }^{4}$ sometimes positional. CVT should be included in the differential of severe, persistent headache of new onset. ${ }^{1,5}$ Other diagnoses to be considered in this type of headache include subarachnoid hemorrhage, intracerebral hemorrhage, arterial dissection, migraine, and reversible vasoconstriction syndrome (RCVS) (-Table 1). Seizures manifest in approximately $10 \%$ of patients with $\mathrm{CVT}^{3}$ due to direct cortical irritation with the involvement of cortical veins and especially if venous infarction and received

January 28, 2019

accepted after revision

April 11, 2019

published online

June 4, 2019
DOI https://doi.org/

10.1055/s-0039-1692023

ISSN 2348-0548.
Copyright $\odot 2019$ Indian Society of Neuroanaesthesiology and Critical Care
License terms

() (1) $\ominus \circledast$ 
hemorrhage occur. Location-dependent, focal neurological signs such as aphasia or motor deficits result if affected areas are anatomically associated with eloquent areas. Raised ICP can arise from multiple mechanisms, particularly decreased or altered venous drainage patterns, mass effect from thrombosis and inflammation, venous infarctions, and resultant intraparenchymal hemorrhage (-Fig. 2). Long-term complication of CVT can be formation of dural arteriovenous fistula (dAVF), due to changes in venous drainage patterns after thrombosis. ${ }^{6}$

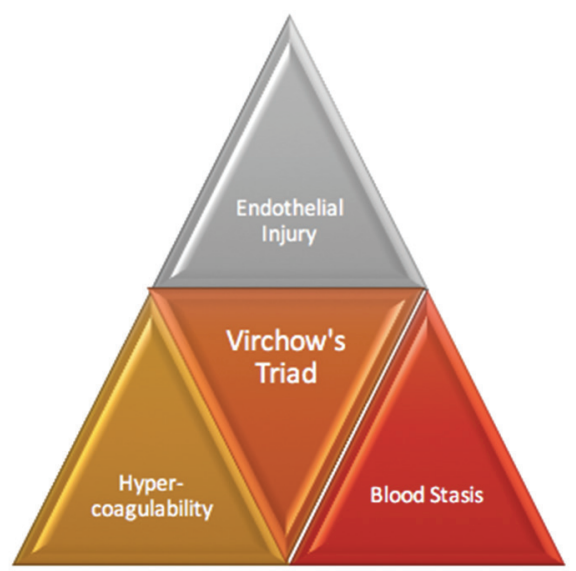

Fig. 1 Virchow's triad.

Table 1 Differential diagnosis for development of "thunderclap" headache

\begin{tabular}{|l|}
\hline Subarachnoid hemorrhage (SAH) \\
\hline Exertion-related headache \\
\hline Migraine \\
\hline Reversible cerebral vasoconstriction syndrome (RCVS) \\
\hline Intracerebral hemorrhage (ICH) \\
\hline $\begin{array}{l}\text { Cerebral venous thrombosis (CVT) } \\
\text { Arterial dissection }\end{array}$ \\
\hline
\end{tabular}

\section{Etiology}

There are numerous causes and risk factors for developing CVT (-Table 2). Heritable hypercoagulable diseases, such as hyper-homocystinemia, antiphospholipid antibody syndrome, and anticardiolipin antibodies, have been implicated in the development of CVT. ${ }^{5}$ Of the more common precipitants, dehydration (poor oral intake, diarrheal illness, etc.) can exacerbate hypercoagulability and provoke CVT in a susceptible patient. Women on oral contraceptives, pregnant or post/peripartum should raise suspicion for this process with concomitant, new-onset/different character of headache due to the relative hypercoagulability of these states. ${ }^{5}$ Angioinvasion of the cavernous sinuses from the facial sinuses due to invasive fungal infections such as mucormycosis are rare but have been described in case reports. ${ }^{7}$ As many of these diseases are more common in women, CVT tends to have a nearly 3:1 gender bias toward women. ${ }^{1}$ Autoimmune diseases, such as Wegener's granulomatosis, Behçet's disease, ${ }^{1}$ and ulcerative colitis, ${ }^{5}$ can also be associated with CVT. Elevated lipoprotein a (Lpa) can be a risk factor for CVT development with a possible fourfold increased risk of recurrence, due to impaired fibrinolysis. ${ }^{8}$

Table 2 Known precipitants of cerebral venous thrombosis formation

\begin{tabular}{|l|}
\hline Female sex \\
\hline Oral contraceptive use \\
\hline Active malignancy \\
\hline Dehydration \\
\hline Young age \\
\hline $\begin{array}{l}\text { Inflammatory diseases (Wegener's granulomatosis, } \\
\text { Behçet's disease, ulcerative colitis) }\end{array}$ \\
\hline $\begin{array}{l}\text { Inherent hypercoagulable states (e.g., hyperhomocystein- } \\
\text { emia, antiphospholipid antibody syndrome) }\end{array}$ \\
\hline $\begin{array}{l}\text { Recent neurosurgical procedure of trauma near the } \\
\text { involved venous structures }\end{array}$ \\
\hline $\begin{array}{l}\text { Elevated lipoprotein a level } \\
\text { Pregnant, peri/antepartum period }\end{array}$ \\
\hline
\end{tabular}
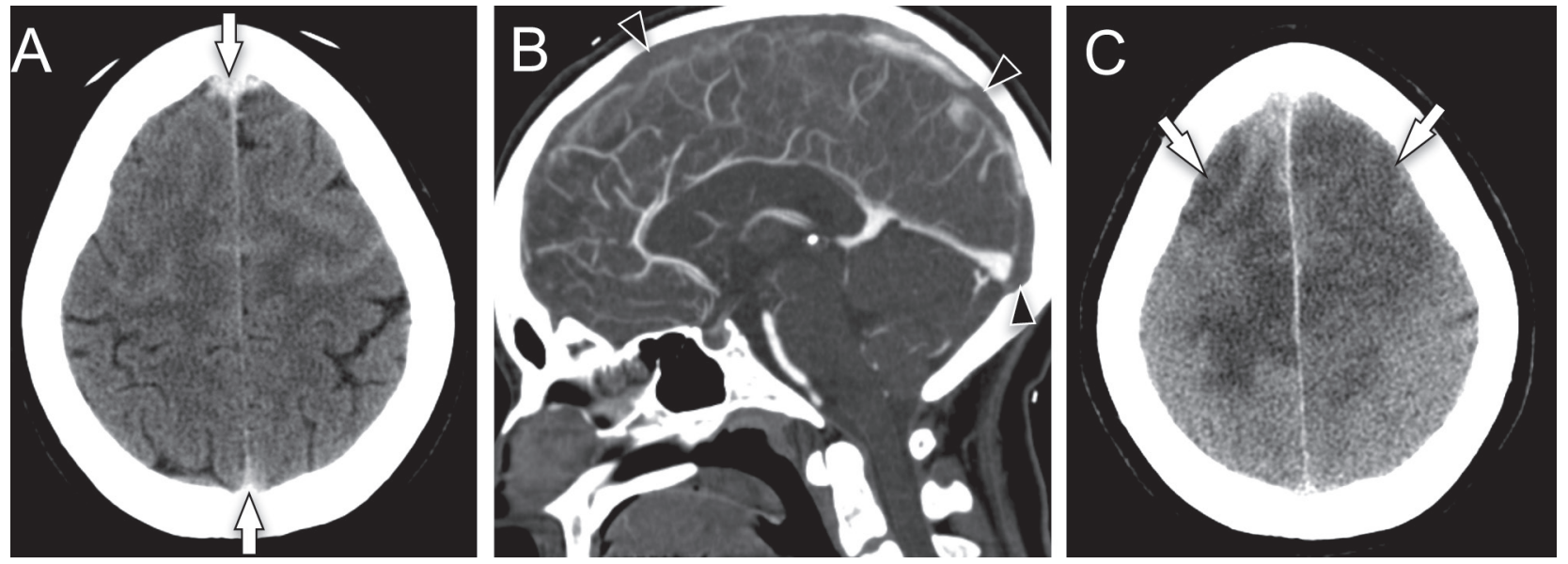

Fig. 2 (A) Axial noncontrast head computed tomography (CT)shows a hyperdense appearance of the superior sagittal sinus (SSS) (white arrows) indicating thrombosis. A small amount of subarachnoid hemorrhage is present in the sulci of the frontal lobes bilaterally. (B) Sagittal image from a CT venogram shows a low-density thrombus filling the SSS (black arrowheads). (C) Axial noncontrast head CT obtained 1 week later shows bifrontal parenchymal hypodensity due to venous infarction (white arrows). 
Recent neurosurgical procedures near the dural sinuses or local trauma are also known precipitants. ${ }^{9}$ CVT should be ruled out when considering a diagnosis of idiopathic intracranial hypertension (IIH) as it can mimic IIH initially. Due to the female predilection for both CVT and IIH, males who are being considered for diagnosis of IIH with signs of ICP such as headache or papilledema, should be evaluated for CVT prior to a diagnosis of IIH being made, as CVT can mimic IIH but has a very obviously different pathology and treatment strategy. ${ }^{1,2}$ CVT should also be suspected in patients with active malignancy presenting with headache and new neurological deficits. The International Study on Cerebral Vein and Dural Sinus Thrombosis (ISCVT) estimated that just over 7\% of cases investigated were associated with active cancer, with thrombosis being caused by malignancy-associated hypercoagulability, direct tumor invasion into venous structures, and/or chemotherapeutic effects. ${ }^{10}$

\section{Diagnosis}

Diagnosis of CVT is made using clinical history that is corroborated with neuroimaging. Dedicated noninvasive techniques that evaluate the patency of the dural venous sinuses include computed tomography venogram (CT Venogram) and magnetic resonance venography (MR venogram). CT venogram provides a high spatial resolution and infrequent technical artifacts but requires intravenous iodinated contrast administration. MR venography can be performed with or without contrast (especially if unable to safely administer this) and provides superior evaluation of the brain parenchyma when paired with magnetic resonance imaging (MRI) of the brain, but it has more frequent technical artifacts. Catheter-based digital subtraction angiography
(DSA) ( - Fig. 3) is less commonly used now due to the ready availability of noninvasive imaging modalities. In small studies, CT venogram was 95\% sensitive and 91\% specific compared with traditional DSA in the detection of CVT. ${ }^{3}$

Diagnosis is made by demonstrating a filling defect or absence of flow in the venous sinuses. On a contrast-enhanced study, there may be central non-enhancing clot in a triangular-shaped venous sinus surrounded by enhancing sinus walls, producing the so-called empty-delta sign.

Additional findings can also be seen on routine brain imaging and may prompt consideration of the diagnosis if not otherwise clinically suspected. On noncontrast head $\mathrm{CT}$, clot in a thrombosed venous sinus can show increased density compared with other venous sinuses, producing the "hyperdense venous sinus" sign (-Fig. 2). ${ }^{11}$ Brain parenchymal hemorrhage is a frequent complication of CVT (-Fig. 3), often involving the parasagittal frontal or parietal lobes, posterior temporal lobes, or thalami. Subarachnoid hemorrhage can also occur. Venous infarction of the parenchyma drained by the occluded sinus may develop, typically beginning in the subcortical white matter and progressing to the overlying cortex.

Involvement of deep venous structures is quite serious, leading to thalamic infarctions with significant clinical deterioration and coma. Thrombosis of the deep venous sinuses as the presenting feature of CVT is more common in infants than in adults. ${ }^{3}$

The D-dimer has a large negative predictive value in thrombotic events. However, measuring a D-dimer has no positive predictive value in CVT diagnosis, as it can be unduly influenced by other acute processes and is an acute phase reactant. Therefore, D-dimer can be used with reasonable
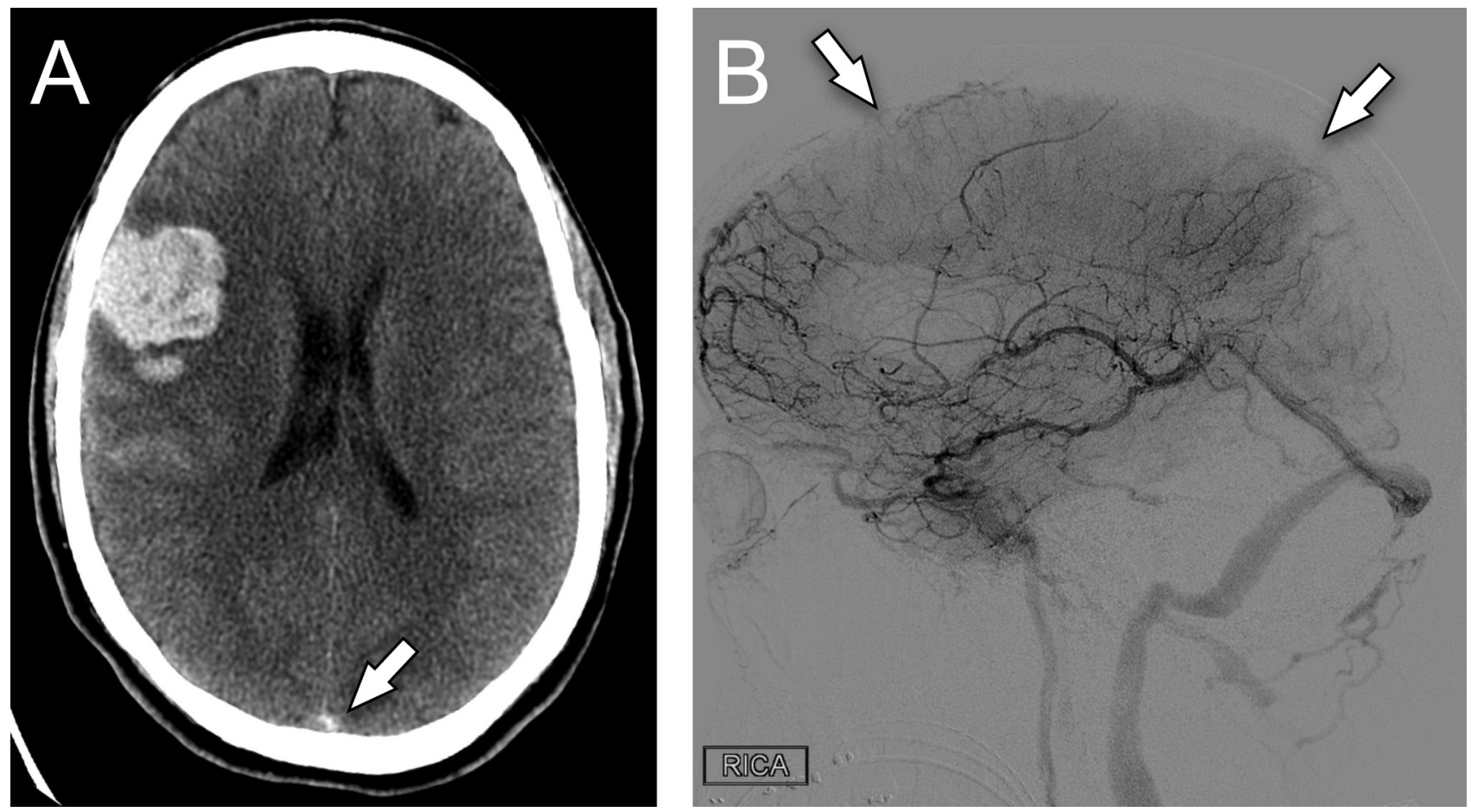

Fig. 3 (A) Axial noncontrast head computed tomography (CT) shows an acute parenchymal hematoma in the right frontal lobe. There is subtle hyperdensity in the superior sagittal sinus (SSS) (white arrow) due to thrombus. (B) Lateral projection of a digital subtraction angiography venogram shows absent filling of the SSS. 
(but not absolute) sensitivity to rule out CVT, but if strong clinical suspicion persists, further workup should be considered. $^{5}$

\section{Treatment}

\section{Standard of Care Medical Therapy}

The standard of care for treatment of acute CVT is systemic anticoagulation, usually with intravenous unfractionated heparin, followed by conversion to oral warfarin. In the presence of venous infarction with intracerebral hemorrhage, anticoagulation remains the mainstay of therapy though its use is counterintuitive. ${ }^{12}$ Intravenous heparinization prevents thrombus propagation and further venous infarction. At present, the American Heart Association/American Stroke Association (AHA/ASA) recommends initiation of systemic intravenous heparinization, low-weight-molecular heparin (LWMH), or ultra-filtrate heparin (UFH), with conversion to oral anticoagulation with the vitamin $\mathrm{K}$ antagonist, warfarin. ${ }^{1,5}$ Duration of treatment is not well defined, but generally a minimum of 3 months of anticoagulation is recommended, with longer courses to be considered for provoked events, and indefinite therapy to be considered in the presence of a known hypercoagulable state. ${ }^{13}$ Increased intracranial pressue (ICP) can arise from multiple mechanisms, particularly decreased or altered venous drainage patterns, mass effect from thrombosis, and/or venous infarctions that may develop as a result. Raised ICP should be treated using standard therapies, including head positioning, hyperosmotic agents, and sedation. ${ }^{13}$ Finally, dAVF is a known complication of CVT, secondary to changes in venous drainage patterns, and may ultimately require surgical intervention. ${ }^{5}$

\section{Invasive Interventional Therapies}

Endovascular therapy is less common, given the relative frailty of venous structures to instrumentation. Indications for endovascular therapy include failure of standard therapy with anticoagulation, worsening neurological deficits, propagation and/or involvement of the deep venous structures, progression to coma, and general risk of death due to neurological decompensation. ${ }^{14}$ To date, surgical approaches to CVT have no proven benefit but require adequately powered and highly rigorous study. Therefore, mechanical thrombectomy is often pursued as a life-saving measure in treatment of refractory cases of CVT. ${ }^{15}$ These patients often have extensive thrombosis affecting the deep venous structures, venous infarctions, and hemorrhage resulting in clinical deterioration to coma. ${ }^{1}$

Decompressive hemicraniectomy may be pursued if resulting intracranial pressure becomes life threatening due to impending herniation with declining neurological examinations into stupor or coma, blown pupils, and signs of ICP not mitigated by medical treatment. In the international study on dural venous and cerebral venous thrombosis (ISCVT study), less than $2 \%$ of the patients studied received this procedure. ${ }^{10,16}$ As a last resort intervention, hemicraniectomy may be a life-saving maneuver but is unlikely to change morbidity of CVT. ${ }^{9,16}$ Systematic analysis of CVT in 2011 published in Stroke ISCVT 2 studied the role of decompressive hemicraniectomy in CVT patients with malignant cerebral edema. Pooled data from 15 studies consisted of patients with poor neurological examination and coma. Sixty-two patients were given decompressive hemicraniectomies (and 17 of these also received hematoma evacuation); 7 received hematoma evacuation only. Of the patients studied, 4 had unfavorable outcomes (modified Rankin scores [mRS] of 4-5) and 11 died, but more than one-half of the patients had an mRS of 3 or less after 12 months. Patients with deep coma and/or bilateral lesions were less likely to have favorable outcomes, despite decompression decreasing the risk of death. ${ }^{16}$

\section{Role of Direct Oral Anticoagulants}

Recognized as noninferior for prevention of other thrombotic events, such as stroke from atrial fibrillation or pulmonary embolism, direct oral anticoagulants (DOACs), such as factor Xa or direct thrombin inhibitors, have not been validated in the treatment of CVT. At present, the European Stroke Organization (ESO) has a recommendation for Coumadin as a treatment for CVT and does not recommend Xa inhibitors; however, it states that this is a weak recommendation with low quality of evidence at present. ${ }^{17}$

A few, small case series of patients with CVT, using recanalization of vessels as a sign of efficacy, have been published. For example, a small case series of 23 patients was reviewed in 2017, which showed no increased rate of new intracranial hemorrhagic events and recanalization in almost all patients, with no significant functional disability in 26 of 27 patients reviewed..$^{18}$ In another example, 236 CVT cases at the Cleveland Clinic were identified between December 2013 and February 2018. In this sample, nine patients were treated with rivaroxaban or apixaban after CVT, though two were initially treated with Coumadin but switched to apixaban and one to rivaroxaban. Within 4 weeks of initiation, patients treated with DOACs were compared with their counterparts who were prescribed standard Lovenox or Coumadin for complications (e.g., gastrointestinal bleeding, inability to maintain therapeutic INR, etc.). None of the nine patients treated with rivaroxaban or apixaban were found to have repeat thrombotic events after 12-month follow-up, and none had clinically significant bleeding. ${ }^{19}$ While promising, significant studies of DOACs in the setting of CVT are necessary to investigate potential roles for this class of medication. Currently, there are several upcoming trials comparing standard warfarin to DOACs, the most prominent being a phase 3 trial called RE-SPECT CVT comparing dabigatran to warfarin for its efficacy in treating CVT, which has reached its primary completion date as of June 2018, and results are pending although preliminarily data suggest that dabigatran is generally as safe as Coumadin. ${ }^{20}$

Patients with CVT and active malignancy are also a target population of interest when it comes to treatment strategies. The standard treatment at this time for malignancy-induced thrombosis is LWMH. ${ }^{21}$ In a case-control study by Silvis et al, they found that history of cancer increased the risk of development of CVT by a factor of five, the most prevalent cancer being hematological malignancy. ${ }^{22}$ At this point of time, there are very little data speaking of the efficacy and safety 
of DOACs for CVT, but several ongoing randomized control trials aimed at looking at their roles in treating thrombotic and thromboembolic events.

\section{Conclusion}

Cerebral venous thrombosis is a dangerous, often underdiagnosed subclass of strokes that requires immediate diagnosis, treatment, and follow-up. While most patients can survive and recover, around $8 \%$ mortality exists after CVT due to complications of cerebral edema, seizures, coma, and herniation. ${ }^{5}$ At this time, heparin and Coumadin remain the standard of care for treatment of CVT. The AHA/ASA is yet to address the potential role of DOAC mediations in CVT treatment, due to lack of outcome data. Due to the efficacy and safety of DOACs for preventing thromboembolism in nonvalvular atrial fibrillation and deep vein thrombosis, ${ }^{23}$ DOAC use after CVT is likely to garner high interest from clinicians. The scientific community should strongly consider adequately powered, rigorous randomized clinical trials to fully investigate the role of DOAC medications in the treatment of CVT. Further study of decompressive craniectomy, as an earlier intervention after CVT, has given reasonable outcomes in otherwise comatose patients with more than one-half of studied patients achieving some functional outcome (varying degrees of recovery, impressively one-third of patients with full recovery with initially fixed and dilated pupils). Finally, as the technology of intravascular catheters continues to progress, endovascular intervention for CVT may prove efficacious. Continued study into the ever-evolving role of newer medications and interventional techniques is necessary to improve patient outcomes after CVT.

\section{Conflict of Interest}

Dr. Dombrowski reports grants from Portola, Eisai Inc., and Sage Therapeutics outside the submitted work. Other authors have no conflicts of interests to declare.

\section{References}

1 Ferro JM, Canhao P. Cerebral Venous Thrombosis: Treatment and Prognosis. Up to Date. Available at: https:// www.uptodate.com/contents/cerebral-venous-thrombosis-treatment-and-prognosis?search=cerebral\%20venous\%20 thrombosis \&source $=$ search_result \&selectedTitle $=2 \sim 15$ 0\&usage_type=default\&display_rank=2. Accessed December 19, 2018

2 Watson T, Shantsila E, Lip GY. Mechanisms of thrombogenesis in atrial fibrillation: Virchow's triad revisited. Lancet 2009;3739658:155-166

3 Yousem DM, Grossman RI. Neuroradiology: The Requisites. 3rd ed. Philadelphia, PA: Mosby Elsevier; 2010

4 Devenney E, Neale H, Forbes RB. A systematic review of causes of sudden and severe headache (thunderclap headache): should lists be evidence based? J Headache Pain 2014;15:49

5 Saposnik G, Barinagarrementeria F, Brown RD Jr, et al; American Heart Association Stroke Council and the Council on Epidemiology and Prevention. Diagnosis and management of cerebral venous thrombosis: a statement for healthcare professionals from the American Heart Association/American Stroke Association. Stroke 2011;42(4):1158-1192
6 Kang MK, Cho YD, Kang HS, Jung KH. Development of a dural arteriovenous fistula subsequent to cerebral venous thrombosis by venous hypertension. eNeurological Sci 2018;14:24-27

7 Gelston CD, Durairaj VD, Simoes EA. Rhino-orbital mucormycosis causing cavernous sinus and internal carotid thrombosis treated with posaconazole. Arch Ophthalmol 2007;125(6):848-849

8 Skuza A, Polak M, Undas A. Elevated lipoprotein(a) as a new risk factor of cerebral venous sinus thrombosis: association with fibrin clot properties. J Thromb Thrombolysis 2019;47(1):8-15

9 Greenberg MS. Handbook of Neurosurgery. 7th ed. New York, NY: Thieme Medical Publishers; 2010

10 Ferro JM, Canhão P, Stam J, Bousser MG, Barinagarrementeria F; ISCVT Investigators. Prognosis of cerebral vein and dural sinus thrombosis: results of the International Study on Cerebral Vein and Dural Sinus Thrombosis (ISCVT) Stroke 2004;35(3):664-670

11 Linn J, Pfefferkorn T, Ivanicova K, et al. Noncontrast CT in deep cerebral venous thrombosis and sinus thrombosis: comparison of its diagnostic value for both entities. AJNR Am J Neuroradiol 2009;30(4):728-735

12 Coutinho JM, Stam J. How to treat cerebral venous and sinus thrombosis. J Thromb Haemost 2010;8(5):877-883

13 Torbey MT, Bösel J, Rhoney DH, et al. Evidence-based guidelines for the management of large hemispheric infarction: a statement for health care professionals from the Neurocritical Care Society and the German Society for Neuro-intensive Care and Emergency Medicine. Neurocrit Care 2015;22(1):146-164

14 Mokin M, Lopes DK, Binning MJ, et al. Endovascular treatment of cerebral venous thrombosis: contemporary multicenter experience. Interv Neuroradiol 2015;21(4):520-526

15 Nimjee SM, Powers CJ, Kolls BJ, Smith T, Britz GW, Zomorodi AR. Endovascular treatment of venous sinus thrombosis: a case report and review of the literature. J Neurointerv Surg 2011;3(1):30-33

16 Ferro JM, Crassard I, Coutinho JM, et al; Second International Study on Cerebral Vein and Dural Sinus Thrombosis (ISCVT 2) Investigators. Decompressive surgery in cerebrovenous thrombosis: a multicenter registry and a systematic review of individual patient data. Stroke 2011;42(10): 2825-2831

17 Ferro JM, Bousser MG, Canhão P, et al; European Stroke Organization. European Stroke Organization guideline for the diagnosis and treatment of cerebral venous thrombosis endorsed by the European Academy of Neurology. Eur J Neurol 2017;24(10):1203-1213

18 Cappellari M, Bovi P. Direct oral anticoagulants in patients with cervical artery dissection and cerebral venous thrombosis. A case series and review of the literature. Int J Cardiol 2017;244:282-284

19 Covut F, Kewan T, Perez O, Flores M, Haddad A, Daw H. Apixaban and rivaroxaban in patients with cerebral venous thrombosis. Thromb Res 2019;173:77-78

20 Ferro JM, Dentali F, Coutinho JM, et al. Rationale, design, and protocol of a randomized controlled trial of the safety and efficacy of dabigatran etexilate versus dose-adjusted warfarin in patients with cerebral venous thrombosis. Int J Stroke 2018;13(7):766-770

21 Piatek C, O'Connell CL, Liebman HA. Treating venous thromboembolism in patients with cancer. Expert Rev Hematol 2012;5(2):201-209

22 Silvis SM, Hiltunen S, Lindgren E, et al. Cancer and risk of cerebral venous thrombosis: a case-control study. J Thromb Haemost 2018;16(1):90-95

23 Lip GYH, Keshishian A, Li X, et al. Effectiveness and safety of oral anticoagulants among nonvalvular atrial fibrillation patients. Stroke 2018;49(12):2933-2944 